ÉGYPTE

monde arabe

\section{Égypte/Monde arabe}

30-31 | 1997

Les visions de l'Occident dans le monde arabe

\title{
Usages et petits usages de « l'Occident » en Égypte
}

Jean-Noël Ferrié

\section{(2) OpenEdition}

\section{Journals}

Édition électronique

URL : https://journals.openedition.org/ema/1663

DOI : 10.4000/ema.1663

ISSN : 2090-7273

\section{Éditeur}

CEDEJ - Centre d'études et de documentation économiques juridiques et sociales

\section{Édition imprimée}

Date de publication : 30 septembre 1997

Pagination : 321-335

ISSN : 1110-5097

\section{Référence électronique}

Jean-Noël Ferrié, « Usages et petits usages de « l'Occident » en Égypte », Égypte/Monde arabe [En ligne], 30-31 | 1997, mis en ligne le 08 juillet 2008, consulté le 07 juillet 2022. URL : http:// journals.openedition.org/ema/1663 ; DOI : https://doi.org/10.4000/ema.1663

Ce document a été généré automatiquement le 7 juillet 2022.

Tous droits réservés 


\title{
Usages et petits usages de « l'Occident » en Égypte
}

\author{
Jean-Noël Ferrié
}

Présenter les usages (et les petits usages) de l'Occident dans la vie quotidienne des Égyptiens requiert au moins deux précautions: ne pas faire état d'une conception «dure» de l'identité, c'est-à-dire ne pas croire que l'Occident existe comme objet « réel » de perception, et ne pas se laisser séduire par l'idée selon laquelle il existerait une « vision » égyptienne de l'Occident. Comme tous les termes servant à dénoter une identité, le mot «Occident » doit, en effet, être compris comme une coquille vide. On ne peut, par exemple, prétendre faire la liste de ses caractéristiques réelles comme on ferait celle des objets contenus dans une pièce. Décrire l'Occident, c'est mettre dans la pièce des objets qui n'y sont pas; ce n'est jamais décrire une réalité composée d'objets déjà là, car les identités sont uniquement des constructions. Ceci est généralement difficile à admettre ou même à penser et le sens commun n'en tient, pour ainsi dire, jamais compte. Il préfère considérer l'identité comme une substance, confondant ainsi l'histoire sociale d'un nom et l'histoire sociale de ses contenus successifs, la transmission d'un signifiant et la transmission d'un signifié. Or l'une n'est pas l'autre. Les contenus successifs d'un nom ou d'un symbole servant à dénoter une identité sont contextuels et ne sont pas liés à la nature du nom ni à la logique de la nomination. Être Bordure, par exemple, ce n'est pas participer d'une identité fixée depuis un millénaire mais c'est toujours être Bordure aujourd'hui et inventer, par rapport à ce présent, l'identité Bordure de jadis.*

Inventer, cela indique aussi que l'on détermine volontairement le contenu de la différence identitaire. En d'autres termes, l'identité n'est pas le constat d'une différence mais la mise en œuvre (et en scène) d'une différenciation. Ainsi les Bordures ne sont-ils pas différents des Syldaves parce qu'ils portent des moustaches mais ils portent des moustaches pour se différencier d'eux. On objectera, certes, que a) les Bordures diffèrent des Syldaves par des traits qu'ils ne revendiquent pas comme distinctifs (ils utilisent de la graisse végétale dans leur cuisine et non de la graisse animale, à l'instar des Syldaves); ou que b) le port des moustaches deviendra une 
différence substantielle durable. Ces deux objections (et toutes celles construites sur ces modèles) ont pour but de soutenir l'existence d'une composante "dure " de l'identité. Mais, si l'on regarde les choses de près, on s'aperçoit que cette identité «dure» n'est jamais revendiquée par les acteurs eux-mêmes qui n'en ont pas conscience ou qui en ont seulement une conscience routinière, partielle et ne prêtant pas à l'engagement. En revanche, elle intéresse les ethnographes et les ethnologues qui construisent des cartes culturelles indiquant, par exemple, où s'arrête la cuisine à l'huile et où commence la cuisine au beurre. À son tour, cette distinction, une fois connue, peut être utilisée par les acteurs comme critère de différenciation. Le fait que les différences réelles ne correspondent pas aux différences utilisées par les processus de différenciations démontre clairement le caractère performatif de l'identité. L'identité est une représentation volontariste de soi. Pour reprendre la distinction formulée par Christian Bromberger (1994) entre «identité performative » et «identité substantielle », l'identité dont on parle est donc toujours l'« identité performative ».

Création consciente (ce qui n'implique ni qu'elle soit rationnelle, ni qu'elle soit seulement instrumentale, ni qu'elle soit libre d'un contenu émotionnel), l'identité n'est pas constituée arbitrairement, même si les critères de différenciations qui la constituent sont arbitraires en ce sens qu'ils ne découlent pas d'une nécessité fonctionnelle. C'est ainsi, par exemple, que les colporteurs musulmans du Népal qui ne différent pas essentiellement des Hindous - se boutonnent à gauche pour se distinguer d'eux (Gaborieau, 1993). En d'autres termes, l'identité se définit toujours par rapport à autrui (Barth, 1969) ; elle est de l'ordre de la différence partagée, c'est-à-dire de la communication. Par communication, il faut simplement entendre qu'il est nécessaire que l'autre dont on se différencie puisse comprendre les termes de la différenciation. Ce dernier point est d'autant plus indispensable que l'étrange étranger est, en fait, plus proche ou que son monde est un monde partagé. Par "monde partagé », j'entends un monde où circulent les mêmes références, les mêmes objets et les mêmes techniques. C'est, bien sûr, le monde décrit quand on évoque la "globalisation ». Certes, il ne faut pas confondre "globalisation » et « homogénéisation », ce dernier terme se bornant à affirmer - d'une façon mélancolique et désabusée - que le monde devient partout pareil. Tout ne devient pas pareil mais les différentes parties du monde finissent par avoir incontestablement un « air de famille». Les performances identitaires ont pour but de publier que cet air de famille est un trompe-1'oeil'1. Elles sont, en ce sens, explicitement formulées afin d'établir (au moins fictivement) l'existence d'entités discrètes là où l'on serait tenté de ne voir qu'un continuum.

4 J'en viens maintenant au second point de cette introduction: l'idée fausse selon laquelle il existerait une vision unique de l'Occident dans les sociétés arabomusulmanes (et, bien sûr, en Égypte). Ce postulat affirme l'existence d'une représentation globale de l'Occident produite par un Orient lui-même global; plus exactement, il se situe dans la perspective de l'existence de deux grands ensembles civilisationnels qui s'opposeraient en tant que tels (mais dont on supposerait néanmoins, d'un point de vue humaniste, qu'ils pourraient s'accorder). Cette perspective implique l'existence d'entités idéelles (la culture arabe, la civilisation musulmane, la civilisation européenne, la culture occidentale...) déterminées par leur origine et régissant, à partir de celle-ci, de vastes configurations sociales. La phrase de Heidegger qui évoque la "tradition bimillénaire de la Métaphysique occidentale " (1959, p.117) peut donner une idée assez exacte des discours tenus dans cette perspective. La tradition se trouve ainsi placée hors de portée des individus; elle met 
en scène des pensées indépendantes de ceux qui les pensent, bien qu'elles contribuent à les façonner. Ce point de vue à la fois déterministe et idéaliste méconnaît que les constructions du monde sont contingentes, historicisées et - surtout - fragmentées, partielles et indéterminées. De plus, elles sont toujours mises en œuvre de façon indirecte, sans que l'on puisse affirmer qu'elle «s'applique». Nous pouvons, par exemple, constater l'existence d'une riche rhétorique égyptienne sur l'altérité de l'Occident (comme il existe en "Occident" une non moins riche rhétorique sur l'altérité de l'« Orient») sans pouvoir, pour autant, en déduire quoi que ce soit de la place exacte de l'Occident dans la vie sociale, les pensées et les conduites quotidiennes des Égyptiens. Paul Veyne a parfaitement rendu compte de cette disjonction entre le monde platonicien des idées et le monde routinier de la vie de tous les jours.

5 En ce sens, la "tradition bimillénaire de la Métaphysique occidentale » n'existe conjointement comme objet de pensée et comme motivation que pour Heidegger, au moment même où il écrit les phrases fascinantes inventant - pour lui et ses lecteurs cette tradition. Autrement dit, les vastes configurations idéelles - comme l'ensemble des formes symboliques - n'existent que dans la mesure où l'on s'en sert, c'est-à-dire conformément à la détermination de ceux qui les utilisent (Wittgenstein, 1953). Il y a ainsi autant d'«Occident» que d'acteurs éprouvant le besoin de faire référence à l'« Occident ». Certes, leur inventivité est en quelque sorte déterminée par les symboles et les rhétoriques disponibles mais ceux-ci doivent alors être considérés comme une ressource plutôt que comme une source. De là vient qu'il ne peut exister une construction intangible dénommée l'« Occident des Orientaux » et, surtout, que l'on ne saurait parler de l'Occident sans parler en même temps des stratégies d'action qui mobilisent cette référence. Ceci ne nous empêche pas, bien sûr, de tenter de mettre un peu d'ordre dans tout cela et de distinguer des "mécanismes» de la référence à l'Occident dans la société égyptienne. Sans ambition typologique, on peut en dénombrer au moins quatre $: 1^{\circ}$ ) les mécanismes qui font référence à l'immoralité de l'Occident, $2^{\circ}$ ) ceux qui font référence à la «macdonaldisation » des sociétés arabomusulmanes, $3^{\circ}$ ) ceux qui usent de $l^{\prime}$ « Occident » comme d'une esthétique locale de Tailleurs et $4^{\circ}$ ) ceux, enfin, qui utilisent l'Occident pour régler une multitude de microinteractions.

\section{L'immoralité de l'Occident}

6 L'idée selon laquelle l'Occident serait «immoral » est un stéréotype établi en Égypte. Évoquant un séjour qu'il fit aux États-Unis, le cheikh d'Al-Azhar disait un jour qu'il " avait vu les étudiants américains faire sur les campus des choses que même les bêtes du désert ne feraient pas ». Ce point de vue - exprimé ici avec emphase et, peut-être, non sans une certaine malice - est suffisamment répandu pour que les sociétés européennes et américaines passent pour des sociétés de licence, où les relations entre les sexes ne sont pas réglées et où les femmes sont faciles. Les journaux, les débats locaux, les (rares) messages prophylactiques concernant le sida, la télévision, entretiennent cette idée avec plus ou moins d'insistance. Cette idée apparaît aussi dans les conversations et les jugements routiniers. Un Égyptien peut ainsi s'inquiéter sérieusement, dans un tête-à-tête, du fait que les Européens pratiqueraient couramment l'inceste. Toutefois, ces stéréotypes que nous considérons volontiers avec une moue désapprobatrice - voire goguenarde - relèvent de deux registres qu'il faut se 
garder de confondre. Le premier de ces registres n'a rien de spécifiquement lié à l'Égypte et aux sociétés arabo-musulmanes. Ainsi, l'idée que les femmes d'autres pays sont, pour l'essentiel, des femmes «culturellement » faciles parce que culturellement différentes, est établie en Europe même et tenue pour une évidence contre l'expérience même qui prouve le contraire ${ }^{2}$. Le stéréotype de naguère faisant des Suédoises des femmes légères et de la Suède un paradis sexuel, devrait ainsi nous familiariser avec le point de vue «oriental» sur les «Occidentales». Il s'agit moins ici d'une vision de l'Occident que d'une instrumentalisation fantasmatique de l'autre.

7 En revanche, beaucoup moins fantasmatique est l'utilisation de l'Occident comme marqueur local d'indignité, lorsqu'on use du terme «occidentalisé(e)» pour désigner un comportement immoral ou, tout au moins, négativement non conventionnel. Quand des Égyptiens disent, par exemple, d'une Marocaine ou d'une Tunisienne qu'elle est occidentalisée, ils veulent signifier qu'elle ne respecte pas les règles de la pudeur et de la modestie féminine, qu'elle n'a pas (selon eux) le comportement normal (pour autant qu'il en existe un) d'une Arabe et d'une musulmane. En même temps, ils définissent indirectement le comportement idéal de la femme arabo-musulmane. Au demeurant, cet adjectif peu flatteur s'appliquera aussi à des Égyptiennes musulmanes ou coptes (Ferrié, à paraître). Dans ce cas, le terme " occidentalisée » a, beaucoup moins que le précédent, un contenu exotique ; son contenu est en fait strictement normatif et dénué de fantaisie ; il a trait à un phénomène considéré comme local et distinctif : la moralité et, plus spécifiquement, la moralité sexuelle des musulmans.

8 Mais, contrairement à l'espoir des moralistes et des partisans de la réislamisation, la moralité sexuelle est beaucoup moins un signe distinctif qu'une façon de se distinguer qui n'a rien de spécifique aux sociétés arabo-musulmanes et qui relève, en fait, d'une stratégie de différenciation d'avec le proche. Le premier point ne demande guère d'explications puisqu'il s'agit quasiment d'une constante anthropologique. Prétendre que l'on possède des mœurs irréprochables alors que celles des voisins seraient déplorables est, en effet, une pratique courante, observable aussi bien dans les sociétés amazoniennes que dans les sociétés européennes (Mosse, 1985). C'est, en quelque sorte, la contrepartie négative de l'idée selon laquelle les autres sociétés seraient des paradis sexuels. Le second point est peut-être plus complexe et nécessite un court détour par l'explication que Peter Brown donne du renoncement sexuel chez les Romains convertis au christianisme. Pour ces convertis qui ne se distinguaient guère, par ailleurs, du restant de la population et ne disposaient pas de rites spectaculaires, l'austérité représentait le seul marqueur identitaire fort qui puisse les constituer en groupe spécifique (Brown, 1995). En d'autres termes, le repli sur les corps, l'investissement surérogatoire dans l'austérité (et peut-être surtout dans sa mise en scène) seraient la conséquence d'une véritable difficulté à se différencier autrement. Il est alors tentant d'interpréter le recours à la morale sexuelle, en tant que moyen de distinction identitaire, comme la conséquence paradoxale de l'imbrication consentie des sociétés arabo-musulmanes dans le monde mis en place par la diffusion des références, des techniques et des objets "occidentaux ». L'avantage d'un tel procédé c'est qu'il permet, tout à la fois, d'instaurer une différence consistante, de la fonder sur des critères généralement reconnus de part et $d^{\prime}$ autre $^{3}$ et - surtout - de le faire dans un domaine qui ne met pas en cause le fonctionnement du reste. Être "pudique " n'empêche ni la circulation des techniques et des objets, ni l'intégration dans un 
système mondial créé et dominé par les « Occidentaux ». Cela n'empêche, en somme, ni la femme de travailler, ni le FMI de régenter, ni le Coca-Cola de pétiller.

\section{La « macdonaldisation » des sociétés orientales}

Il faudrait, cependant, se garder d'en déduire que le Coca-Cola ou le MacDonald's - qui ne semblent pas toucher à la moralité - sont unanimement acceptés. Ce n'est pas le cas et il en va de même pour la plupart des produits originaires "d'Occident ", dès lors qu'ils peuvent être perçus comme transportant un système de référence non pas seulement alternatif mais concurrent. Prenons un exemple: le fait pour des groupes mixtes d'adolescents égyptiens d'aller ensemble au MacDonald's peut être considéré comme une conduite ludique ne prêtant pas à mal ni même à commentaire. Toutefois, un «petit entrepreneur de morale» - pour suivre l'heureuse expression de Howard Becker (1985)- peut très bien faire remarquer que cette sorte de loisir favorise une mixité contraire à la moralité publique et, bien sûr, à la morale musulmane. Il s'attaquera ainsi à des pratiques «occidentales » apparemment innocentes - aller au MacDonald's - qui entraînent d'autres pratiques qui ne le sont pas.

Remarquons maintenant que cette formulation qui use d'une rhétorique relativiste peut servir à dissimuler efficacement les motivations moralisatrices du petit entrepreneur. Par "relativisme», on entend la conception selon laquelle toutes les «cultures » ont un droit égal à la reconnaissance, ce droit invalidant toute approche critique du contenu d'une culture. Ainsi que l'a remarqué Richard Rorty (1991), la position relativiste est essentiellement "occidentale » dans sa genèse, mais elle s'est largement diffusée de sorte qu'elle est devenue une ressource rhétorique partagée entre les «Occidentaux» et les "Orientaux». Cette conception permet de critiquer l'invasion des produits "occidentaux» au nom du respect des cultures locales considérées non plus comme des systèmes normatifs (que l'on pourrait toujours mettre en cause) mais comme des patrimoines, ce qui aboutit à l'euphémisation de leur contenu normatif. On préfère, en somme, poser le problème en termes de respect des différences. D'une façon générale, ce type d'argumentation est très répandu et débute toujours par l'affirmation d'une spécificité culturelle, indiscutable dans son fondement. Grâce à cette rhétorique, peuvent être mises en cause des choses aussi hétérogènes que la démocratie, le cinéma étranger, les droits de l'homme ou les variétés européennes ${ }^{4}$.

11 Je voudrais insister, maintenant, sur le fait que le mécanisme de cette mise en cause est le même, qu'elle soit motivée par un point de vue religieux et moralisateur ou non, qu'elle porte sur des sujets "graves» ou sur des sujets «futiles». Prenons, par exemple, un sujet "grave»: la «démocratie ». L'argument relativiste consistera à affirmer : « Nous sommes des musulmans, la démocratie ce n'est pas pour nous; elle est pour les Occidentaux parce qu'elle permet tout. Elle a permis la sexualité, la mixité. La démocratie est dissolution des mœurs. $»^{5}$. Ici, la mise en cause de la "démocratie » se fonde sur son extranéité. L'argument moral qui suit, n'est pas déterminant en ce sens que la même affirmation : "La démocratie n'est pas pour nous ", peut être suivie par des considérations politiques: «Nous les Arabes, ce qu'il nous faut c'est un pouvoir fort ", ou sociologiques : « Nous sommes trop indisciplinés ». Revenons, à présent, sur le MacDonald's. J'ai donné la version moralisatrice : «Cela favorise la mixité », mais il faut aussi donner la version scientifique: «Cette nourriture n'est pas conforme au climat égyptien» et la version sociologique: «Cette façon de manger détruit la 
sociabilité égyptienne, elle est caractéristique d'une société individualiste $»^{6}$. Ainsi, la condamnation de "produits » de "l'Occident » peut-elle s'attacher à des productions relevant de mondes différents - ici, le monde du politique et celui des loisirs - et en dénoncer la nocivité au nom de raisons différentes qui ne sont pas cumulables. En d'autres termes, elles ne dépeignent pas un "Occident» unique mais autant « d'Occidents » que de points de vue sur les produits qui y sont référés.

La critique de la «macdonaldisation» de l'Égypte ne se confond donc ni avec une condamnation globale de l'Occident ni avec la volonté d'imposer un ordre moral fondé sur l'islam. Par " macdonaldisation », j'entends le point de vue critique selon lequel les produits occidentaux mais, plus particulièrement, les produits américains (entendus au sens large) coloniseraient le monde et imposeraient (ou tenteraient d'imposer) une société et une culture identiques. Ce point de vue critique est argumenté à partir de conceptions contrastées de ce qui est bon ou mauvais pour les sociétés « orientales». Ces conceptions ne font pas appel aux mêmes critères d'évaluation. Si la critique de la mixité au MacDonald's et dans les sociétés démocratiques se réfère explicitement au registre religieux, la critique du même MacDonald's comme destructeur des sociabilités traditionnelles renvoie, certes, explicitement à une mise en cause de «l'individualisme occidental » mais ne relève pas d'un registre religieux ou simplement moral. Elle ne relève même pas, d'ailleurs, d'un registre que l'on pourrait identifier comme proprement «oriental». Les mêmes considérations sur les nouveaux modes de consommation, les aspects individualistes, «non conviviaux» de certains d'entre eux, sont également présentes dans les sociétés « occidentales ». Certes, dans l'ensemble des propos sur la "macdonaldisation ", on peut -bien évidemment- repérer tout une série de critiques qui ont un "air de famille", même si elles portent sur des choses très différentes. Le propos de l'étudiante sur la démocratie en est un exemple. Mais ces propos s'insèrent dans d'autres qui ne participent pas de cet «air de famille ». De plus, l'argument relativiste «ce n'est pas pour nous » sur lequel se fondent les attaques contre la «macdonaldisation » peut, ainsi que je l'ai déjà indiqué, exprimer quelque chose qui ressemble plus à de la nostalgie qu'à une condamnation.

\section{L'Occident décoratif}

13 Si l'on quitte, maintenant, le domaine discursif pour s'intéresser aux occurrences de «l'Occident» dans le décor et dans les pratiques de la vie quotidienne, on s'apercevra qu'elles y occupent une place non négligeable, laquelle n'est pas ouverte à la contestation. Mais, sans doute, faut-il ici distinguer deux choses, afin d'éviter une confusion assez habituelle. Le terme "occurrences de l'Occident » ne désigne pas les références, les objets et les techniques en provenance de ce que l'on nomme « les pays occidentaux ». D'une certaine manière, les sociétés « orientales » sont construites par ceux-ci dans des proportions qui dépassent largement la simple importation de techniques nécessaires. Cette chose-là est admise, routinière et ne se prête guère à des discours qui la prendraient directement comme objet (même si elle constitue l'arrièreplan de maints discours sur la revivification de l'islam ou des nations arabes). En fait, le terme "occurrences de l'Occident» désigne toutes les occasions où l'Occident est simplement le nom d'un imaginaire local de « railleurs », c'est-à-dire d'un «ailleurs » faisant partie du local lui-même. 
14 Je m'explique. Nous serons tous prêts à admettre qu'une femme voilée qui va, en compagnie de son mari, dîner dans un restaurant de spécialités mexicaines du Caire appartenant à une chaîne américaine (et dont la gérante est elle-même américaine), n'a pas le moins du monde le sentiment de pénétrer dans un lieu voué à l'altérité. Pour elle, il s'agit d'un lieu agréable (sauf si la cuisine est mauvaise) de la routine ludique cairote. Cet aspect routinier est bien sûr ce qui domine ; toutefois, ici, la routine n'implique pas - comme c'est le cas avec les téléviseurs, les machines à laver, les vestes croisées et les pantalons fuseau - d'oublier la provenance des choses. Bien au contraire, celle-ci fait partie de leurs propriétés recherchées et de leur signification locale. Les objets décorant le restaurant et renvoyant à l'Amérique des années cinquante, les photographies montrant des rituels festifs strictement américains, les tables carrelées, ce Mexique de fantaisie inventé aux États-Unis pour être importé en des lieux totalement étrangers, tous ces aspects participent, en fait, à la fabrication d'un « ailleurs » proportionné au monde égyptien. Durant le jeûne du mois de Ramadan, on y vient manger au moment de la rupture et l'on y fume aussi la shîsha ${ }^{7}$ comme dans des restaurants de spécialités «traditionnelles» égyptiennes. En d'autres termes, «Tailleurs » est débarrassé de son altérité tout en étant néanmoins toujours conçu comme tel.

Une infinité de choses de la vie quotidienne offre ainsi une vision non polémique de Tailleurs occidental: les parfums, les bouteilles de Coca-Cola ou de Pepsi-Cola, les feuilletons étrangers passant à la télévision égyptienne, les films américains, les marques de voiture et de vêtement (Lacoste, Benetton...), les noms français donnés à des salons de thé égyptiens (Palais de l'Élysée, La Poire...), les noms anglais des chaînes de restauration rapide, les personnages de dessins animés comme Tom et Jerry, les cookies, les marques de cigarettes et leur publicité... Certes, toutes ces choses sont virtuellement des moyens de distinction identitaire et un petit entrepreneur de morale peut aisément les tirer de leur routine pour en faire des objets de polémique, mais il en sera de même de n'importe quel objet puisque la volonté d'ouvrir une polémique précède le choix de la cause. Cependant, la quasi-totalité des objets de Tailleurs demeurent d'un usage routinier; ils n'en construisent pas moins une acception locale de «l'Occident» bien différente de celles des polémistes et, en définitive, tout aussi efficace.

\section{L'Occident comme moyen de régler des « problèmes tout à fait secondaires »}

Je voudrais en venir pour finir à un usage, certes, incontestablement polémique de "l'Occident» mais néanmoins dégagé de toute intention normative d'ensemble, puisqu'il ne vise qu'à régler des situations personnelles. Susan Ossman (à paraître) a mis en évidence ce type de situations, en indiquant qu'elles se caractérisaient par l'affirmation d'une différence identitaire pour mettre fin à une interaction. Prenons un exemple : une Égyptienne discute avec une amie française ; à un moment donné, elles ont un point de désaccord; l'Égyptienne dit: «Tu es une Occidentale, tu ne peux pas comprendre ». Cette affirmation règle une discorde en bloquant un processus argumentatif par l'insertion d'une différence indicible. Évidemment, on peut croire qu'une différence de cette sorte existe véritablement. Toutefois, ce serait méconnaître que la personne qui use d'un pareil argument en use bien souvent là où une autre - qui 
ne pourrait se prétendre d'une culture différente - devrait trouver un autre moyen pour rompre la conversation à son avantage. Car, il ne faut pas perdre de vue que, dans ce genre de situation, le contenu du reproche ( tu as tort de penser ainsi, de me contredire, de faire ça ») est indépendant de la forme qu'il prend (« tu penses comme ça parce que tu es occidental»).

Si l'on voulait ainsi rassembler toutes les phrases entendues qui insistent sur une différence culturelle, à l'occasion d'une discorde, on remarquerait que leur contenu varie en fonction de la discorde elle-même, et que les défauts de "l'Occident " se confondent avec la liste infinie des griefs liés aux divergences entre les interactants. Ces « problèmes tout à fait secondaires " peuvent sembler totalement anecdotiques. Ils ne le sont toutefois pas, dans la mesure où nous avons une certaine tendance à penser, quand nous entendons des propos comme : «Il dit cela parce que c'est un Occidental» ou, pire encore, quand nous en sommes le destinataire, que l'auteur de ce jugement coupant est animé par tout un arrière-plan de représentations critiques vis-à-vis de l'Occident, qu'il est en quelque sorte le porte-parole d'un " état d'esprit ». Cet excès d'imagination de notre part nous conduit à inventer des visions globales là où rien de tel ne se montre. Le plus souvent notre interlocuteur ne parle que pour lui et non pour son frère ou l'un des siens (et encore moins pour tous les siens). Dans des discussions avec des collègues arabes, il m'est arrivé de me voir objecter : "Vous pensez comme ça parce que vous êtes un Occidental», et de me sentir alors injustement disqualifié comme contradicteur. Je remâchais ma colère (consécutive à la déconvenue de ne pouvoir répliquer valablement dans le même registre) en réfléchissant à la structure du système de pensée qui pouvait amener des gens sains d'esprit à croire que le fait d'être (éventuellement) autre qu'eux m'empêchait de parler des mêmes choses qu'eux. Mais je me serais sans doute évité bien des peines, si j'avais seulement compris que mes interlocuteurs usaient davantage d'un moyen pour faire valoir leur point de vue qu'ils n'exposaient toute une philosophie.

\section{Du face à face des « civilisations » au monde partagé}

Les quatre mécanismes de référence à «l'Occident» dans les sociétés arabomusulmanes, que je viens assez rapidement de décrire, ont sans doute très peu à voir avec cette chose inquiétante et majestueuse que l'on nomme " la vision de l'Occident ". Il est possible de s'en étonner, comme quand on ne retrouve pas des choses familières dans un lieu que l'on croyait connaître. Mon propos vise précisément à cela, à dire que ces êtres familiers n'existent pas, qu'il n'y a pas une «vision de l'Occident» dont on pourrait disserter, évaluer l'influence sur les relations internationales et sur l'évolution des sociétés qui la produisent. Certes, on peut toujours isoler des discours, rechercher dans les oeuvres édifiantes et chez les polémistes de quoi montrer qu'il existe une conception globale de «l'Occident » et que cette conception est globalement partagée en « Orient ». Mais, ce faisant, nous nous bornons à sélectionner un discours parmi une infinité d'autres ; et cette sélection nous renseigne davantage sur ce que nous pensons, nous, des autres que sur ce que les autres pensent de nous.

Toutefois, ce serait se tromper de problème que de poser les choses en termes de bonnes ou de mauvaises intentions. Je crois plutôt que nous avons affaire à une erreur dans l'ontologie même des protagonistes. Pour toute une série de raisons, nous sommes en effet enclins à penser qu'il existe des ensembles culturels distincts, dotés de 
spécificités fortes et que ces ensembles culturels se pensent réciproquement, à partir de leurs traditions respectives. Si nous adoptons un point de vue humaniste (mais ce n'est pas forcément le cas), nous nommons ces ensembles des "civilisations » et appelons à leur dialogue. Nous supposons ainsi l'existence de frontières délimitant des visions du monde et des visions d'autrui. Ce faisant, nous sommes très loin du monde tel qu'il est et dont la principale caractéristique est d'être un monde partagé. Cela ne veut pas dire un même monde, cela veut dire simplement que les oppositions ne suivent pas le tracé des frontières politiques ou des hypothétiques frontières civilisationnelles. Des références, des objets et des techniques de "l'Occident » font partie de la routine des sociétés arabo-musulmanes, et l'on pourrait dire - de ce point de vue - qu'elles sont inscrites dans le même espace que les sociétés dites occidentales. D'autres références, d'autres objets et d'autres techniques sont, en revanche, impliqués dans des discours de dénonciation, mais ces discours - comme j'ai essayé de le montrer - ne sont pas forcément convergents. De plus, la même référence - la démocratie, par exemple - peut donner lieu à des discours favorables comme à des discours dévaforables et il n'y a nulle raison de penser que les uns sont plus représentatifs ou plus structurants que les autres. Il s'agit d'usages et de petits usages qui s'insèrent dans la trame de la vie quotidienne. Ils ne la tissent pas.

\section{BIBLIOGRAPHIE}

BARTH F., 1969, Ethnie Groups and Boundaries, Boston, Little, Brown and Co. BECKER H., 1985, Outsiders, Paris, Métaillé.

BOETSCH G., FERRIE J.-N. :

-1993, « La Mauresque aux seins nus. L'imaginaire erotique de la carte postale coloniale », dans BLANCHARD P., CHATELIER A., Images et Colonies, Paris, Syros.

-1995, « Contre Alloula : le Harem colonial revisité », dans BEAUGÉ G., L'Image dans le Monde arabe, Paris, CNRS.

BROMBERGER C, 1994, «L'ethnologie de la France et le problème de l'identité », Civilisation, vol. XLII, $\mathrm{n}^{\circ} 2$.

BROWN P., 1995, Le Renoncement à la chair. Virginité, célibat et continence dans le christianisme primitif, Paris, Gallimard.

FERRIÉ J.-N., à paraître, «Qu'est-ce qu'un copte selon les musulmans ? Construction de la frontière et typification de soi », DÉCOBERT C, Dynamiques identitaires en Égypte.

GABORIEAU M., 1993, Ni brahmanes ni ancêtres. Colporteurs musulmans du Népal, Paris, Presses de l'EHESS.

HEIDEGGER M., 1959, Qu'appelle-t-on penser, Paris, PUF.

MOSSEG., 1985, Nationalism and Sexuality. Respectability and Abnormal Sexuality in Modem Europe, New-York, Howard Fertig. 
OSSMANS., à paraître, « Bob Marley entre nous deux ? », dans WINKIN Y, La Communication interculturelle.

RADIS. :

- 1995a, La Réislamisation de la société égyptienne vue par les étudiants de l'Université du Caire, Rapport à la Fondation Ford, Le Caire.

-1995b, « Pour une approche anthropologique de la réislamisation », Egypte/Monde arabe, n 24.

RORTY R., 1991, «Ethnocentrism: A Reply to Geertz »in RORTY R. (ed.), Objectify, Relativism and Thruth. Philosophical Papers, vol. 1, Cambridge, Cambridge University Press.

WITTGENSTEIN, 1953, Philosophical Investigation, Oxford, Blackwell.

\section{NOTES}

* Il est bien évidemment fait ici référence aux personnages de la bande dessinée d'Hergé, Le sceptre d'Ottokar, N.D.L.R.

1. Je ne me préoccuperai pas ici des motivations qui pousse des acteurs à affirmer une distinction identitaire, mais il doit être clair que celles-ci sont loin de se limiter au « souci d'être soi ». Les enjeux de pouvoir me paraissent bien plus déterminants, l'identité servant à légitimer des positions, des statuts et des privilèges.

2. Ce que montre très bien l'imaginaire de la "mauresque au sein nu », c'est-à-dire l'imaginaire mis en scène par ces innombrables cartes postales produites durant toute la période coloniale et où l'on voyait des «femmes indigènes" plus ou moins nues. Les photographies donnaient à croire que le Maroc, la Tunisie et l'Algérie étaient un paradis sexuel. Sur cet imaginaire, voir Boëtsch et Ferrié (1993 et 1995).

3. Ceci est bien sûr la conséquence du fait que ce critère de distinction est quasiment universel. Il est néanmoins diversement interprété. Le moralisme sexuel par lequel les sociétés musulmanes entendent se distinguer, apparaît, bien souvent, rétrograde aux « Occidentaux » et responsable d'une mise en minorité de la femme. Ce conflit d'interprétation ne peut que favoriser l'établissement d'une frontière identitaire, puisqu'il y a accord sur les termes de l'opposition.

4. Précisons immédiatement que cette mise en cause n'est pas forcément faite d'un point de vue musulman (comme on pourrait facilement être amené à le croire) mais aussi d'un point de vue «arabe», les coptes (ou, plus largement, les chrétiens d'Orient) pouvant parfaitement critiquer «l'occidentalisation des mœurs".

5. Déclaration d'une étudiante en $2^{\mathrm{e}}$ année de science politique à l'Université du Caire. Cité et commenté dans S. Radi (1995a et 1995b).

6. Cette conception fut exprimée - presque dans les mêmes termes - dans un journal égyptien durant l'automne 1995.

7. Pipe à eau, plus connue en Europe sous le nom de narguilé.

\section{INDEX}

Mots-clés : identité, Occident 
AUTEUR

JEAN-NOËL FERRIÉ

Cedej 